\title{
Is loss of smell an early predictor of COVID-19 severity: a systematic review and meta-analysis
}

\author{
Sujata Purja ${ }^{1}$ Hocheol Shin ${ }^{1} \cdot$ Ji-Yun Lee ${ }^{2}$. \\ EunYoung Kim $^{1,3}$ (D)
}

Received: 16 June 2021 / Accepted: 17 July 2021 / Published online: 24 July 2021

(C) The Pharmaceutical Society of Korea 2021

\begin{abstract}
Anecdotal evidence suggests that the severity of coronavirus disease of 2019 (COVID-19), caused by severe acute respiratory syndrome coronavirus 2 (SARSCoV-2), is likely to be distinguished by variations in loss of smell (LOS). Thus, we conducted a meta-analysis of 45 articles that include a total of 42,120 COVID-19 patients from 17 different countries to demonstrate that severely ill or hospitalized COVID-19 patients have a lesser chance of experiencing LOS than non-severely ill or non-hospitalized COVID-19 patients (odds ratio $=0.527$ [95\% CI $0.373-0.744 ; p<0.001]$ and 0.283 [95\% CI $0.173-0.462$; $p<0.001]$, respectively). We also proposed a possible mechanism underlying the association of COVID-19 severity with anosmia, which may explain why patients without sense of smell develop severe COVID-19. Variations in LOS according to the severity of COVID-19 is a global phenomenon, with few exceptions. Since severely ill patients have a lower rate of anosmia, patients without anosmia should be
\end{abstract}

Supplementary Information The online version contains supplementary material available at https://doi.org/10.1007/ s12272-021-01344-4.

EunYoung Kim

eykimjcb777@cau.ac.kr

1 Evidence-Based Research Laboratory, Department of Health, Social and Clinical Pharmacy, College of Pharmacy, Chung-Ang University, 84 Heukseok-ro, Dongjak-gu, Seoul 06974, South Korea

2 Pathophysiology Laboratory, College of Pharmacy, Chung-Ang University, 84 Heukseok-ro, Dongjak-gu, Seoul 06974, South Korea

3 Clinical Research Laboratory College of Pharmacy, Division of Licensing of Medicines and Regulatory Science, The Graduate School of Pharmaceutical Management, Chung-Ang University, Seoul, Republic of Korea monitored more closely in the early stages of COVID-19, for early diagnosis of severity of illness. An understanding of how the severity of COVID-19 infection and LOS are associated has profound implications for the clinical management and mitigation strategies for the disease.

Keywords COVID-19 - SARS-CoV-2 · Anosmia · COVID-19 severity $\cdot$ COVID-19 hospitalization

\section{Introduction}

Despite the efforts of rapidly distributing the coronavirus disease (COVID-19) vaccine, various severe acute respiratory syndrome coronavirus 2 (SARS-CoV-2) genetic variants have emerged and are spreading quickly worldwide with deadly outcomes. Few experts believe that the COVID-19 pandemic would last longer than expected (Boehm et al. 2021). Thus, there is a need for early recognition of COVID-19 disease and disease severity for the rational management of the pandemic. The clinical manifestations of SARS-CoV-2 infection range from asymptomatic infections to severe neurological complications (Gandhi et al. 2020a). Acute respiratory syndrome with non-specific signs or symptoms caused by COVID-19 are fever, cough, chills, dyspnea, myalgia, and sore throat (Baj et al. 2020). Also, patients with COVID-19 may experience a period of chemosensory disorders including anosmia without any other symptoms early in the disease (Meng et al. 2020a). These disorders are more frequently observed in females and younger patients and less frequently in patients who smoke or have co-morbidities (Talavera et al. 2020). Olfactory dysfunctions (OD) are widespread in COVID-19 and olfactory recovery could take from one week to more than one month with different patterns of recovery (Amer et al. 2020). On the 
other hand, the prevalence of OD in COVID-19 patients was significantly higher with objective evaluation than subjective measurements (Hannum et al. 2020).

Chemosensory disorders can be used as a diagnostic marker for early COVID-19 disease (Liang et al. 2020). Early detection for chemosensory disorders, including anosmia, in non-severely ill or otherwise asymptomatic individuals, may be a helpful strategy to prevent transmission of the initial stage of the disease. A study reported that patients with anosmia have a lower mortality rate and intensive care unit (ICU) admission (Talavera et al. 2020). Similarly, olfaction complaints were reported more in patients with mild flu syndrome than in patients with severe flu syndrome (Mendonça et al. 2021). Some previous systematic reviews and meta-analyses demonstrated the prevalence and various relevant factors that affect chemosensory dysfunction in COVID-19 (Aziz et al. 2021; Hajikhani et al. 2020; Tong et al. 2020; von Bartheld et al. 2020). Although these reviews are fairly comprehensive, have early initial data, and are current, they still have remained uncertain to evaluate OD objectively and with classification of COVID-19 severity. Thus, a meta-analysis including recently updated and objective olfactory evaluation data is required to identify a clear association between COVID-19 severity and anosmia.

Our objective was to examine the association between COVID-19 severity and loss of smell (LOS) in patients with COVID-19. We also proposed a possible mechanism behind the correlation between COVID-19 severity and LOS, which may explain why patients without anosmia may develop severe forms of COVID-19.

\section{Materials and methods}

\section{Data sources}

To identify eligible studies published until February 28, 2021, the COVID-19 portfolio of the National Institute of Health (NIH) (https://icite.od.nih.gov/covid19/search/) was searched using the keywords, "anosmia," "olfactory disorders," and "loss of smell." This site offers a detailed, expert-curated source of NIH publications and preprints pertaining either to COVID-19 or SARS-CoV-2, with current data that are updated regularly. Thus, grey literatures are also included in our analysis. Additionally, we conducted a second search of databases such as PubMed, EMBASE and NIH COVID-19 portfolio from Dec 2019 to June 2021 with various related search terms to ensure the comprehensive literature search on the related topic and to add newly published studies. The search term and search strategy applied for the literature search are provided in the Supplementary Table S1 and S2.

\section{Eligibility criteria}

Any articles in English that were novel reports of LOS in patients with confirmed COVID-19 were included. We excluded studies that did not report quantitative data, review articles, studies with a small sample size ( $\leq 10$ patients), conference abstracts, letter to the editor, randomized controlled trial studies and cohorts where patients were not differentiated as hospitalized and non-hospitalized or as severe and non-severe. When COVID-19 severity was not classified in the respective studies, severe COVID-19 patients were defined as patients requiring intensive care or those who died and non-severe COVID-19 patients were defined as patients requiring no intensive care, or those who were alive. Furthermore, when studies classified COVID-19 severity as mild, moderate, and severe, we excluded the total number of participants with moderate disease to ensure that severe and non-severe patients were separated consistently throughout the study. This meta-analysis only included studies that reported anosmia as a separate event; any studies that targeted patients with olfactory and/or gustatory disorders were excluded.

\section{Selection process}

Records were managed in EndNote version 20. Studies were screened by two independent researchers (S.P. and E.Y.) based on titles and abstracts. Studies that reported anosmia in the confirmed COVID-19 patients were advanced to the second round of screening. Full texts were reviewed using prespecified selection criteria. Any disagreement between the two authors was addressed through discussion. 


\section{Data extraction and collection}

The following categories of information were obtained: first author's name; date of publication; type of study, the country where the study was performed, sex distribution, number of confirmed patients in each classified groups, and number of patients with LOS. When a study included both subjective self-reporting and objective olfactory evaluation data, we collected objective olfactory evaluation data for the analysis to obtain accurate and objective data on LOS. We included the peer-reviewed version when preprints were published in a peer-reviewed journal.

\section{Assessment of bias}

The quality assessment of the included studies was conducted according to the checklist provided by the Newcastle-Ottawa Scale (NOS) (Wells et al. 2021) for cohort and case control studies, whereas for cross-sectional studies, Joanna Briggs Institute (JBI) was used (Moola et al. 2020).
Publication bias was evaluated by examining the funnel plot and Egger's test (Egger et al. 1997). A $p$ value $<0.05$ was considered statistically significant.

\section{Data synthesis}

All statistical analyses were conducted using the Comprehensive Meta-Analysis (Biostat, Englewood, NJ, USA) program. We estimated the odds ratio (OR) with a 95\% confidence interval (CI) for OD in severely ill versus non-severely ill patients with COVID-19 and inpatients versus outpatients with COVID-19, according to the weighted pooled average effect measures based on the size and precision of each study. Both meta-analyses were two-sided, testing the null hypothesis that the estimated OR was 1 . We also conducted a sensitivity analysis with published studies excluding grey literatures to compare and analyse the OR of LOS. Heterogeneity within the study was calculated by the $I^{2}$ statistic (Higgins et al. 2003). Random-effect models were used because heterogeneity existed among the included studies.

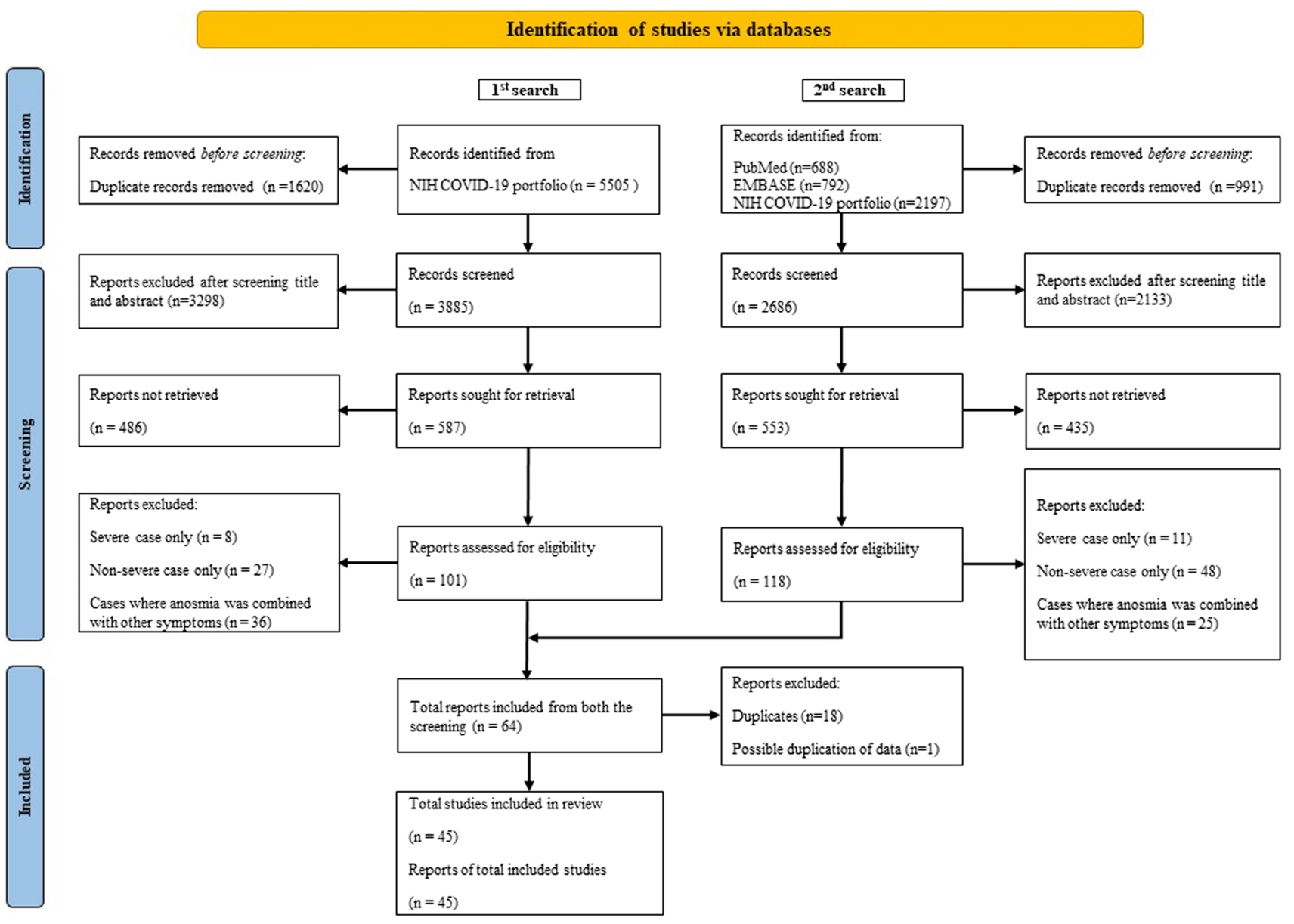

Fig. 1 Flowchart of study selection in accordance with PRISMA guidelines. In total, first and second comprehensive search of databases provided 45 studies involving confirmed cases according to severity and hospitalization status with respect to anosmia 


\section{Results}

\section{Study selection}

In total, we identified 5505 studies through first literature search (3885 after removal of duplicates), including preprints that had not yet been peer-reviewed. After screening by title and abstracts, 3298 studies were excluded and 101 studies were fully reviewed, of which 30 met the inclusion criteria. While our second search provided 3677 papers (2686 after removal of duplicates), including preprints before peer review; 2133 studies were excluded after screening by title and abstracts; 118 studies were fully reviewed, of which 34 met the inclusion criteria. After removing the duplicates that met the inclusion criteria from two searches, 45 studies were included in the final analysis (Fig. 1).

\section{Study characteristics}

Our study comprised 39 cohorts, 5 cross-sectional studies, and one case-control study. Publication years ranged from April 2020 to June 2021. The studies were conducted in Iran $(n=7)$, Spain $(n=6)$, United States $(n=4)$, France $(n=4)$, Italy $(n=4)$, China $(n=3)$, Turkey $(n=3)$, Nigeria $(n=2)$, Germany $(n=2)$, India $(n=2)$, United Kingdom $(n=1)$, Ireland $(n=1)$, Oman $(n=1)$, Brazil $(n=1)$, Chile $(n=1)$, Greece $(n=1)$, Georgia $(n=1)$, and one study conducted in two countries (Belgium and France). Of the 45 included studies, 34 studies were focused on severely ill versus non-severely ill patients, and 11 studies were focused on hospitalized versus non-hospitalized patients. In total, there were five preprints and 40 peer-reviewed studies. A total of 42,120 patients (5349 severe patients, 32,009 nonsevere patients, 2241 hospitalized patients, and 2521 nonhospitalized patients) with COVID-19 from 45 studies were included in the analysis. Table1 shows the characteristics of the included studies.

\section{Assessment of bias}

For assessing the risk of bias within cohort and case-control studies, we used the NOS method, and all included studies were of moderate to high quality, with scores over 6 (Supplementary Table 3 and 4). For assessing the risk of bias within cross-sectional studies, we used JBI method, and all studies were included in our analysis (Supplementary Table 5). Inspection of funnel plots visually showed no apparent asymmetry for the analyses of OR for disease severity (Fig. 2a) or hospitalization (Fig. 2b). Egger's test did not show publication bias in our analyses of disease severity or hospitalization ( $p=0.548$ and $p=0.184$, respectively).

\section{Association between COVID-19 severity and LOS}

In total, 34 studies provided information on the severity of COVID-19 disease. Of them, four studies were preprints (Al Harthi et al. 2020; Bertlich et al. 2020; Papizadeh et al. 2020; Patel et al. 2020), and 30 articles were published studies (Aggarwal et al. 2020; Alasia et al. 2021; Alizadehsani et al. 2021; Allenbach et al. 2020; Amanat et al. 2021; Borobia et al. 2020; Delorme et al. 2021; Elimian et al. 2020; Ermis et al. 2021; García-Azorín et al. 2021; Ghaffari et al. 2021; Goyal et al. 2021; Izquierdo et al. 2020; KadianeOussou et al. 2020; Kocayı ğıt et al. 2021; Lechien et al. 2021; Liotta et al. 2020; Mao et al. 2020; McElvaney et al. 2020; Muñoz-Rodríguez et al. 2021; Printza et al. 2021; Romero-Sánchez et al. 2020; Salepci et al. 2021; Sobhani et al. 20212021; Song et al. 2021; Studart-Neto et al. 2020; Sun et al. 2021; Tomlins et al. 2020; Vaira et al. 2020; Vial et al. 2020). The OD were present in 482 severely ill and 2640 non-severely ill patients with COVID-19. The association between COVID-19 severity and smell disorder was statistically significant with an OR of 0.527 (95\% CI $0.373-0.744 ; p<0.001$ ), suggesting that loss of smell was less frequent in severely ill patients (Fig. 3). In a sensitivity analysis using published studies alone, the OR was 0.478 (95\% CI $0.344-0.665 ; p<0.001)$ (Supplementary Fig. S1).

\section{Association between COVID-19 hospitalization and LOS}

Our meta-analysis comprises a total of 11 reports that included data on the hospitalization status of patients. Of them, one was a preprint (Zobairy et al 2020), and the others 
Table 1 Characteristics of the included Studies

\begin{tabular}{|c|c|c|c|c|c|c|c|c|}
\hline Study & Study site & Peer reviewed & $\begin{array}{l}\text { Olfactory assess- } \\
\text { ment method }\end{array}$ & Study design & Total patients & Total females & Total males & Total LOS \\
\hline \multicolumn{9}{|c|}{ Severity of COVID-19 disease (severe versus non-severe) } \\
\hline $\begin{array}{l}\text { Aggarwal } \\
\text { (2020) May }\end{array}$ & US & Yes & $\begin{array}{l}\text { Retrospective } \\
\text { data collection } \\
\text { from medical } \\
\text { record }\end{array}$ & Cohort & 16 & 4 & 12 & 3 \\
\hline $\begin{array}{l}\text { Al Harthi } \\
\text { (2020) Jul }\end{array}$ & Oman & No & $\begin{array}{l}\text { Retrospective } \\
\text { data collection } \\
\text { from medical } \\
\text { record }\end{array}$ & Cohort & 102 & 23 & 79 & 7 \\
\hline $\begin{array}{l}\text { Alasia (2021) } \\
\text { Jan }\end{array}$ & Nigeria & Yes & $\begin{array}{l}\text { Retrospective } \\
\text { data collection } \\
\text { from medical } \\
\text { record }\end{array}$ & Cohort & 646 & 172 & 474 & 75 \\
\hline $\begin{array}{l}\text { Alizadehsani } \\
\text { (2021) April }\end{array}$ & Iran & Yes & Descriptive & Cohort & 123 & 61 & 62 & 33 \\
\hline $\begin{array}{l}\text { Allenbach } \\
(2020) \text { Oct }\end{array}$ & France & Yes & $\begin{array}{l}\text { Retrospective } \\
\text { data collection } \\
\text { from medical } \\
\text { record }\end{array}$ & Cohort & $150^{\mathrm{a}}$ & - & - & 16 \\
\hline $\begin{array}{l}\text { Amanat (2021) } \\
\text { Mar }\end{array}$ & Iran & Yes & Self-reported & Cohort & 873 & 317 & 556 & 561 \\
\hline $\begin{array}{l}\text { Bertlich (2020) } \\
\text { May }\end{array}$ & Germany & No & $\begin{array}{l}\text { SNOT-22 ques- } \\
\text { tionnaire and } \\
\text { BSIT }\end{array}$ & Cohort & 47 & 13 & 34 & 14 \\
\hline $\begin{array}{l}\text { Borobia (2020) } \\
\text { Jun }\end{array}$ & Spain & Yes & $\begin{array}{l}\text { Retrospective } \\
\text { data collection } \\
\text { from medical } \\
\text { record }\end{array}$ & Cohort & 2226 & 1152 & 1074 & 284 \\
\hline $\begin{array}{l}\text { Delorme } \\
\text { (2021) Jun }\end{array}$ & France & Yes & $\begin{array}{l}\text { Retrospective } \\
\text { data collection } \\
\text { from medical } \\
\text { record }\end{array}$ & Cohort & 244 & 97 & 147 & 39 \\
\hline $\begin{array}{l}\text { Elimian (2020) } \\
\text { Dec }\end{array}$ & Nigeria & Yes & $\begin{array}{l}\text { Retrospective } \\
\text { data collection } \\
\text { from medical } \\
\text { record }\end{array}$ & Cohort & $3215^{\mathrm{a}}$ & - & - & 23 \\
\hline $\begin{array}{l}\text { Ermis (2021) } \\
\text { Mar }\end{array}$ & Germany & Yes & $\begin{array}{l}\text { Retrospective } \\
\text { data collection } \\
\text { from medical } \\
\text { record }\end{array}$ & Cohort & 53 & 21 & 32 & 14 \\
\hline $\begin{array}{l}\text { García-Azorín } \\
\text { (2021) Apr }\end{array}$ & Spain & Yes & Self-reported & Cohort & $206^{\mathrm{b}}$ & - & - & 36 \\
\hline $\begin{array}{l}\text { Ghaffari (2021) } \\
\text { Jan }\end{array}$ & Iran & Yes & Questionnaire & Cohort & 361 & 147 & 214 & 69 \\
\hline $\begin{array}{l}\text { Goyal (2021) } \\
\text { Jun }\end{array}$ & India & Yes & Questionnaire & Cohort & $398^{\mathrm{c}}$ & - & - & 163 \\
\hline $\begin{array}{l}\text { Izquierdo } \\
\text { (2020) Oct }\end{array}$ & Spain & Yes & $\begin{array}{l}\text { Retrospective } \\
\text { data collection } \\
\text { from medical } \\
\text { record }\end{array}$ & Cohort & 10,504 & - & - & 300 \\
\hline $\begin{array}{l}\text { Kadiane-Ous- } \\
\text { sou (2020) } \\
\text { Nov-Dec }\end{array}$ & France & Yes & $\begin{array}{l}\text { Retrospective } \\
\text { data collection } \\
\text { from medical } \\
\text { record }\end{array}$ & Cohort & 114 & 48 & 66 & 54 \\
\hline
\end{tabular}


Table 1 (continued)

\begin{tabular}{|c|c|c|c|c|c|c|c|c|}
\hline Study & Study site & Peer reviewed & $\begin{array}{l}\text { Olfactory assess- } \\
\text { ment method }\end{array}$ & Study design & Total patients & Total females & Total males & Total LOS \\
\hline $\begin{array}{l}\text { Kocayığıt } \\
\text { (2021) Apr }\end{array}$ & Turkey & Yes & $\begin{array}{l}\text { Retrospective } \\
\text { data collection } \\
\text { from medical } \\
\text { record }\end{array}$ & Cohort & 82 & 36 & 46 & 14 \\
\hline $\begin{array}{l}\text { Lechien (2021) } \\
\text { Jan }\end{array}$ & $\begin{array}{l}\text { Belgium and } \\
\text { France }\end{array}$ & Yes & $\begin{array}{l}\text { Sniffin-sticks } \\
\text { and SNOT-22 } \\
\text { method }\end{array}$ & Cohort & $233^{\mathrm{d}}$ & 154 & 79 & 118 \\
\hline $\begin{array}{l}\text { Liotta }(2020) \\
\text { Nov }\end{array}$ & US & Yes & $\begin{array}{l}\text { Retrospective } \\
\text { data collection } \\
\text { from medical } \\
\text { record }\end{array}$ & Cohort & 509 & - & - & 58 \\
\hline $\begin{array}{l}\text { Mao (2020) } \\
\text { Apr }\end{array}$ & China & Yes & Subjective & Cohort & 214 & 127 & 87 & 11 \\
\hline $\begin{array}{l}\text { Mcelvaney } \\
\text { (2020) Sep }\end{array}$ & Ireland & Yes & Descriptive & Cohort & 40 & 15 & 25 & 7 \\
\hline $\begin{array}{l}\text { Muñoz-Rod- } \\
\text { ríguez (2021) } \\
\text { Apr }\end{array}$ & Spain & Yes & $\begin{array}{l}\text { Retrospective } \\
\text { data collection } \\
\text { from medical } \\
\text { record }\end{array}$ & Cohort & 12,126 & 5667 & 6359 & 653 \\
\hline $\begin{array}{l}\text { Papizadeh } \\
\text { (2020) }\end{array}$ & Iran & No & $\begin{array}{l}\text { Retrospective } \\
\text { data collection } \\
\text { from medical } \\
\text { record }\end{array}$ & Cohort & 186 & 88 & 98 & 44 \\
\hline $\begin{array}{l}\text { Patel (2020) } \\
\text { oct }\end{array}$ & India & No & $\begin{array}{l}\text { Retrospective } \\
\text { data collection } \\
\text { from medical } \\
\text { record }\end{array}$ & Cohort & 549 & 151 & 398 & 22 \\
\hline $\begin{array}{l}\text { Printza (2021) } \\
\text { Mar }\end{array}$ & Greece & Yes & Phone interview & Cohort & $90^{c}$ & - & - & 34 \\
\hline $\begin{array}{l}\text { Romero- } \\
\text { Sánchez } \\
\text { (2020) Aug }\end{array}$ & Spain & Yes & $\begin{array}{l}\text { Retrospective } \\
\text { data collection } \\
\text { from medical } \\
\text { record }\end{array}$ & Cohort & 841 & 368 & 473 & 41 \\
\hline $\begin{array}{l}\text { Salepci (2021) } \\
\text { Feb }\end{array}$ & Turkey & Yes & Interview & Cross-sectional & 223 & 110 & 113 & 71 \\
\hline $\begin{array}{l}\text { Sobhani (2021) } \\
\text { Feb }\end{array}$ & Iran & Yes & Interview & Cohort & 397 & 174 & 223 & 29 \\
\hline $\begin{array}{l}\text { Song (2021) } \\
\text { Feb }\end{array}$ & China & Yes & $\begin{array}{l}\text { Data collected } \\
\text { from medical } \\
\text { record and } \\
\text { reevaluated by } \\
\text { phone inter- } \\
\text { view }\end{array}$ & Cohort & 1172 & 595 & 577 & 134 \\
\hline $\begin{array}{l}\text { Studart-Neto } \\
\text { (2020) Aug }\end{array}$ & Brazil & Yes & $\begin{array}{l}\text { Retrospective } \\
\text { data collection } \\
\text { from medical } \\
\text { record }\end{array}$ & Cohort & 89 & 34 & 55 & 8 \\
\hline $\begin{array}{l}\text { Sun }(2021) \\
\text { May }\end{array}$ & China & Yes & $\begin{array}{l}\text { Data collected } \\
\text { from medical } \\
\text { record and } \\
\text { reevaluated by } \\
\text { phone inter- } \\
\text { view }\end{array}$ & Cohort & 932 & 557 & 375 & 29 \\
\hline $\begin{array}{l}\text { Tomlins (2020) } \\
\text { Aug }\end{array}$ & UK & Yes & $\begin{array}{l}\text { Retrospective } \\
\text { data collection } \\
\text { from medical } \\
\text { record }\end{array}$ & Cohort & 95 & 35 & 60 & 3 \\
\hline
\end{tabular}


Table 1 (continued)

\begin{tabular}{|c|c|c|c|c|c|c|c|c|}
\hline Study & Study site & Peer reviewed & $\begin{array}{l}\text { Olfactory assess- } \\
\text { ment method }\end{array}$ & Study design & Total patients & Total females & Total males & Total LOS \\
\hline $\begin{array}{l}\text { Vaira (2020) } \\
\text { July }\end{array}$ & Italy & Yes & CCCRC & Cohort & $220^{\mathrm{c}}$ & - & - & 148 \\
\hline $\begin{array}{l}\text { Vial (2020) } \\
\text { Dec }\end{array}$ & Chile & Yes & $\begin{array}{l}\text { Retrospective } \\
\text { data collection } \\
\text { from medical } \\
\text { record }\end{array}$ & Cohort & $88^{\mathrm{e}}$ & 45 & 43 & 7 \\
\hline \multicolumn{9}{|c|}{ Status of hospitalization (inpatients versus outpatients) } \\
\hline $\begin{array}{l}\text { Avc1 et al. } \\
\text { (2020) Aug }\end{array}$ & Turkey & Yes & $\begin{array}{l}\text { Retrospective } \\
\text { data collection } \\
\text { from medical } \\
\text { record }\end{array}$ & Cohort & 1197 & 497 & 700 & 529 \\
\hline $\begin{array}{l}\text { Bakhshaee } \\
\text { (2021) May }\end{array}$ & Iran & Yes & Subjective & Cohort & 502 & - & - & 173 \\
\hline $\begin{array}{l}\text { Bianco (2021) } \\
\text { April }\end{array}$ & Italy & Yes & Self-reported & Cross-sectional & 50 & 21 & 29 & 26 \\
\hline $\begin{array}{l}\text { D'Ascanio } \\
\text { (2021) Jan }\end{array}$ & Italy & Yes & Questionnaire & Case-control & 43 & 14 & 29 & 26 \\
\hline $\begin{array}{l}\text { Izquierdo- } \\
\text { Domínguez } \\
\text { (2020) June }\end{array}$ & Spain & Yes & Questionnaire & Cross-sectional & 846 & 400 & 446 & 454 \\
\hline $\begin{array}{l}\text { Killerby (2020) } \\
\text { Jun }\end{array}$ & Georgia & Yes & $\begin{array}{l}\text { Retrospective } \\
\text { data collection } \\
\text { from medical } \\
\text { record }\end{array}$ & Cohort & 531 & 303 & 228 & 134 \\
\hline $\begin{array}{l}\text { Nouchi (2021) } \\
\text { Oct }\end{array}$ & France & Yes & Interview & Cross-sectional & 390 & 188 & 202 & 129 \\
\hline $\begin{array}{l}\text { Paderno (2020) } \\
\text { Aug }\end{array}$ & Italy & Yes & Questionnaire & Cross-sectional & 508 & 223 & 285 & 283 \\
\hline $\begin{array}{l}\text { Vahey (2021) } \\
\text { Feb }\end{array}$ & US & Yes & Phone interview & Cohort & 364 & 176 & 187 & 176 \\
\hline $\begin{array}{l}\text { Yan (2020) } \\
\text { July }\end{array}$ & US & Yes & Self-reported & Cohort & 128 & 67 & 61 & 75 \\
\hline $\begin{array}{l}\text { Zobairy (2020) } \\
\text { July }\end{array}$ & Iran & No & Questionnaire & Cohort & 203 & 91 & 112 & 25 \\
\hline
\end{tabular}

Note When included studies did not classify COVID-19 severity, severe patients were defined as patients requiring intensive care or those who died, and non-severe patients were patients requiring no intensive care or patients who were alive

Dashes denote numbers unstated in the source

LOS loss of smell, CCCRC clinical research center orthonasal olfaction test, SNOT-22 sino-nasal outcome tool-22, BSIT brief smell identification test

${ }^{\mathrm{a}}$ Total sample size with anosmia status

${ }^{\mathrm{b}}$ Information about the severity of COVID-19 was available for 206 patients

${ }^{\mathrm{c}}$ Moderate sample size was excluded

${ }^{\mathrm{d}}$ Sample size of the objective olfactory evaluation was included

${ }^{\mathrm{e} O n l y}$ inpatients sample size was included since anosmia was present as a separate variable 

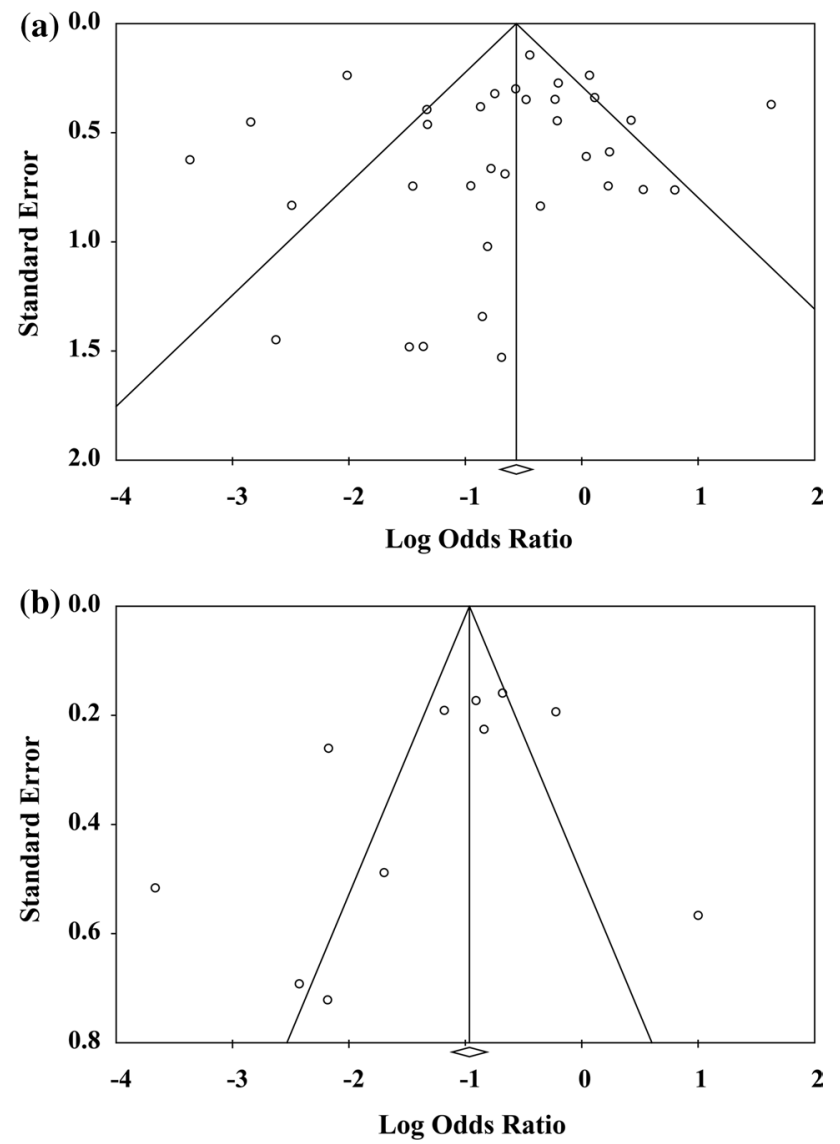

Fig. 2 Funnel plots representing effect estimates and standard errors of each report in the meta-analysis. Funnel plots for estimated a odds ratio for the association between COVID-19 severity and smell disorder and $\mathbf{b}$ odds ratio for the association between hospitalization of patients with COVID-19 and smell disorder. The white circle represents values for reports included in each analysis. The sides of the triangle illustrate the expected inverted funnel shape

were published studies (Avc1 et al. 2020; Bakhshaee et al. 2021; Bianco et al. 2021; D’Ascanio et al. 2021; Izquierdo-Domínguez et al. 2020; Killerby et al. 2020; Nouchi et al. 2021; Paderno et al. 2020; Vahey et al. 2021; Yan et al. 2020). The LOS was present in 735 inpatients and 1295 outpatients. The OR was 0.283 (95\% CI 0.173-0.462, $p<0.001)$, indicating that the incidence of OD is lower among hospitalized patients (Fig. 4). The sensitivity analysis without grey literatures showed an odds ratio of 0.240 (95\% CI 0.148-0.389, $p<0.001)$ (Supplementary Fig. S2).

\section{Discussion}

In this systematic review and meta-analysis, we included 45 studies involving a total of 42,120 COVID-19 patients evaluating the association of COVID-19 severity or hospitalization status with LOS, which showed that the odds of LOS were significantly lower in severely ill or hospitalized patients than in non-severely ill or non-hospitalized patients (OR 0.527 [95\% CI 0.373-0.744; $p<0.001$ ] and OR 0.283 [95\% CI $0.173-0.462 ; p<0.001]$, respectively). In comparison to previous systematic reviews and meta-analyses (Aziz et al. 2021; Hajikhani et al. 2020; Tong et al. 2020; von Bartheld et al. 2020), we evaluated the association of COVID-19 severity with LOS, excluding gustatory dysfunction and combined disorders. To the best of our knowledge, our study is the first large-scale analysis to report the association between COVID-19 severity (mild verse severe forms) and LOS.

A subgroup analysis reported that as the number of hospitalized patients in a study increased, LOS became less common $(b=-0.019, p<0.001)$ (von Bartheld et al. 2020). Similarly, another study reported that the odds of patients with severe COVID-19 disease and LOS were significantly lower when compared to patients with severe COVID-19 disease and without $\operatorname{LOS}(\mathrm{OR}=0.36$, CI $0.27-0.48 ; p<0.01)$ (Aziz et al. 2021). Similar to these findings, we found that severe or hospitalized COVID-19 patients are less likely to experience LOS than non-severe or non-hospitalized COVID-19 patients.

The precise location and extent of the damage caused by SARS-CoV-2 in OD are not well known. The nose is exposed to the outer environment and is involved in several innate defence responses. Consequently, a portion of the heterogeneity in the clinical status of COVID-19 may be affected by the fluctuation of nasal infectivity driven by the environment (Wu et al. 2020). The event of chemosensory disorders in patients with mild COVID-19 underpins the hypothesis that early infection and active replication occurs in the upper respiratory tract, followed by subsequent aspiration to the lower lung that leads to severe illness (Wölfel et al. 2020). Recognition of the virus in the nasal cavity activates the immune system, which then recruits the cytokines and other inflammatory mediators that can initiate an antiviral response within the nasal epithelium (Rodriguez et al. 2020; Sepahi et al. 2019). These cytokines can cause apoptosis of olfactory receptor neurons (ORN) or damage 


\begin{tabular}{|c|c|c|c|c|c|c|c|c|}
\hline \multirow{2}{*}{ Study } & LOS & $\begin{array}{l}\text { Severe } \\
\text { atients } \\
\text { Total }\end{array}$ & LOS & $\begin{array}{r}\text { Non } \\
\text { Severe } \\
\text { atients } \\
\text { Total }\end{array}$ & \multirow{2}{*}{ Weight } & \multirow{2}{*}{$\begin{array}{c}\text { Odds Ratio } \\
\text { Random, 95\% CI }\end{array}$} & \multirow{2}{*}{\multicolumn{2}{|c|}{$\begin{array}{c}\text { Odds Ratio } \\
\text { Random, 95\% CI }\end{array}$}} \\
\hline & & 10tal & LUS & lotal & & & & \\
\hline Aggarwal 2020 & 1 & 8 & 2 & 8 & $1.25 \%$ & $0.429[0.031,5.985]$ & & \\
\hline Al Harthi 2020 & 0 & 19 & 7 & 82 & $1.08 \%$ & $0.258[0.014,4.718]$ & & \\
\hline Alasia 2021 & 6 & 61 & 69 & 585 & $3.51 \%$ & $0.816[0.339,1,966]$ & & \\
\hline Alizadehsani 2021 & 0 & 15 & 33 & 108 & $1.11 \%$ & $0.073[0.004,1.251]$ & & \\
\hline Allenbach 2020 & 3 & 46 & 13 & 99 & $2.75 \%$ & $0.462[0.125,1.707]$ & & \\
\hline Amanat 2021 & 172 & 299 & 389 & 574 & $4.40 \%$ & $0.644[0.483,0.859]$ & 1. & \\
\hline Bertlich 2020 & 4 & 9 & 10 & 38 & $2.45 \%$ & $2.240[0.500,10.039]$ & & \\
\hline Borobia 2020 & 5 & 460 & 279 & 1766 & $3.49 \%$ & $0.059[0.024,0.143]$ & $-1-$ & \\
\hline Delorme 2021 & 18 & 124 & 21 & 120 & $3.85 \%$ & $0.801[0.403,1.591]$ & & \\
\hline Elimian 2020 & 1 & 295 & 22 & 2920 & $1.79 \%$ & $0.448[0.060,3.336]$ & & \\
\hline Ermis 2021 & 2 & 28 & 12 & 25 & $2.25 \%$ & $0.083[0.016,0.429]$ & ——- & \\
\hline Garcia-Azorin 2021 & 3 & 126 & 33 & 80 & $2.88 \%$ & $0.035[0.010,0.119]$ & $-1-$ & \\
\hline Ghaffari 2021 & 42 & 233 & 27 & 128 & $4.09 \%$ & $0.823[0.479,1.412]$ & & \\
\hline Goyal 2021 & 18 & 60 & 145 & 338 & $4.01 \%$ & $0.570[0.315,1.032]$ & & \\
\hline Izquierdo 2020 & 3 & 83 & 297 & 10421 & $3.01 \%$ & $1.278[0.401,4.071]$ & & \\
\hline Kadiane-Oussou 2020 & 17 & 55 & 37 & 59 & $3.69 \%$ & $0.266[0.122,0.579]$ & & \\
\hline Kocayigit 2021 & 9 & 52 & 5 & 30 & $2.93 \%$ & $1.047[0.315,3.472]$ & & \\
\hline Lechien 2021 & 19 & 52 & 99 & 181 & $3.94 \%$ & $0.477[0.253,0.901]$ & & \\
\hline Liotta 2020 & 11 & 134 & 47 & 375 & $3.85 \%$ & $0.624[0.314,1.242]$ & & \\
\hline Mao 2020 & 3 & 88 & 8 & 126 & $2.67 \%$ & $0.521[0.134,2.020]$ & & \\
\hline McElvaney 2020 & 3 & 20 & 4 & 20 & $2.24 \%$ & $0.706[0.136,3.658]$ & & \\
\hline Munoz-Rodriguez 2021 & 18 & 2020 & 635 & 10106 & $4.19 \%$ & $0.134[0.084,0.215]$ & $-1-$ & \\
\hline Papizadeh 2020 & 23 & 48 & 21 & 138 & $3.77 \%$ & $5.126[2.464,10.662]$ & & $-1-$ \\
\hline Patel 2020 & 2 & 159 & 20 & 390 & $2.50 \%$ & $0.236[0.054,1.020]$ & & \\
\hline Printza 2021 & 15 & 34 & 19 & 56 & $3.52 \%$ & $1.537[0.641,3.685]$ & & \\
\hline Romero-Sanchez 2020 & 9 & 329 & 32 & 512 & $3.74 \%$ & $0.422[0.199,0.896]$ & & \\
\hline Salepci 2021 & 6 & 45 & 65 & 178 & $3.45 \%$ & $0.267[0.107,0.666]$ & & \\
\hline Sobhani 2021 & 2 & 61 & 27 & 336 & $2.50 \%$ & $0.388[0.090,1.676]$ & & \\
\hline Song 2021 & 24 & 199 & 110 & 973 & $4.19 \%$ & $1.076[0.672,1.722]$ & & \\
\hline Studart-Neto 2020 & 5 & 45 & 3 & 44 & $2.45 \%$ & $1.708[0.383,7.627]$ & & \\
\hline Sun 2020 & 2 & 52 & 27 & 880 & $2.50 \%$ & $1.264[0.292,5.465]$ & & \\
\hline Tomlins 2020 & 0 & 20 & 3 & 75 & $1.02 \%$ & $0.505[0.025,10.183]$ & & \\
\hline Vaira 2020 & 36 & 52 & 112 & 168 & $3.88 \%$ & $1.125[0.575,2.200]$ & & \\
\hline Vial 2020 & 0 & 18 & 7 & 70 & $1.08 \%$ & $0.229[0.012,4.197]$ & & \\
\hline Total $(95 \% \mathrm{CI})$ & & 5349 & & 32009 & $100.0 \%$ & $0.527[0.373,0.744]$ & $>$ & \\
\hline Total events & 482 & & 2640 & & & & 1 & \\
\hline Heterogenetiy: $\mathrm{Tau}^{2}=0$ & 0.684 & $h i^{2}=1$ & $231, \mathrm{~d}$ & $=33$, & 79.659 & & 0.01 & 1 \\
\hline Test for overall effect : 2 & $Z=-$ & $38(p<$ & 001) & & & & Severe & Non \\
\hline
\end{tabular}

Fig. 3 Severely ill patients with COVID-19 are associated with a significantly lower risk of smell disorder. The table summarizes the number of patients with loss of smell (LOS) and the total number of confirmed COVID-19 cases who were either severe or non-severe from 34 reports. For each analysis (grey boxes), the forest plot shows the estimated odds ratio (OR) for the association of LOS with severe COVID-19 cases, with a 95\% confidence interval (CI; horizontal black lines). The estimated pooled OR (grey diamond) was 0.527 (95\% CI 0.373-0.744), which was significantly different from $1(p<0.001)$, according to a two-sided test. A random-effects model was used to calculate effects and summaries 


\begin{tabular}{|c|c|c|c|c|c|c|}
\hline \multirow[b]{2}{*}{ Study } & \multicolumn{2}{|c|}{ Inpatients } & \multicolumn{2}{|c|}{ Outpatients } & \multicolumn{2}{|r|}{ Odds Ratio } \\
\hline & LOS & Total & LOS & Total & Weight & Random, 95\% CI \\
\hline Avc1 2020 & 67 & 215 & 462 & 982 & $11.11 \%$ & $0.510[0.372,0.698]$ \\
\hline Bakhshaee 2021 & 106 & 324 & 67 & 178 & $10.87 \%$ & $0.806[0.550,1.180]$ \\
\hline Bianco 2021 & 8 & 28 & 18 & 22 & $6.15 \%$ & $0.089[0.023,0.346]$ \\
\hline D'Ascanio 2021 & 7 & 20 & 19 & 23 & $5.91 \%$ & $0.113[0.027,0.467$ \\
\hline Izquierdo-Dominguez 2020 & 316 & 649 & 138 & 197 & $11.02 \%$ & $0.406[0.288,0.571]$ \\
\hline Killerby 2020 & 4 & 220 & 130 & 311 & $7.77 \%$ & $0.026[0.009,0.071]$ \\
\hline Nouchi 2020 & 24 & 198 & 105 & 192 & $10.33 \%$ & $0.114[0.068,0.191]$ \\
\hline Paderno 2020 & 130 & 295 & 153 & 213 & $10.89 \%$ & $0.309[0.212,0.450$ \\
\hline Vahey 2021 & 45 & 128 & 131 & 236 & $10.62 \%$ & $0.435[0.279,0.678]$ \\
\hline Yan 2020 & 7 & 26 & 68 & 102 & $8.05 \%$ & $0.184[0.071,0.481]$ \\
\hline Zobairy 2020 & 21 & 138 & 4 & 65 & $7.28 \%$ & $2.737[0.899,8.332]$ \\
\hline Total $(95 \% \mathrm{CI})$ & & 2241 & & 2521 & $100.0 \%$ & $0.283[0.173,0.462]$ \\
\hline $\begin{array}{l}\text { Total events } \\
\text { Heterogeneity : }\end{array}$ & $\begin{array}{l}735 \\
\mathrm{Chi}^{2}\end{array}$ & 48, & $\begin{array}{l}1295 \\
0, I^{2}=\end{array}$ & & & \\
\hline
\end{tabular}

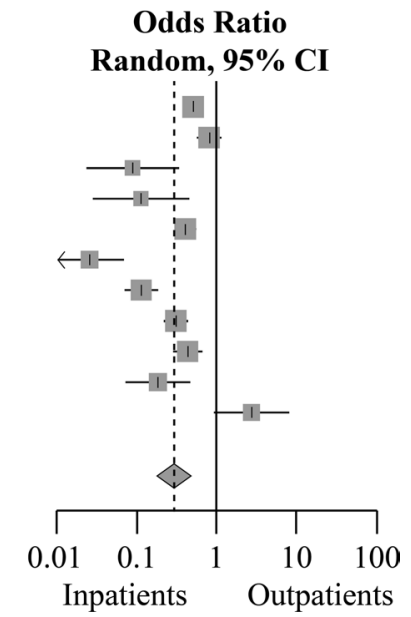

Fig. 4 Patients hospitalized with COVID-19 are associated with a significantly lower risk of smell disorder. The table summarizes the number of patients with loss of smell (LOS) and the total number of confirmed COVID-19 cases who were either hospitalized or non-hospitalized from 11 reports. The forest plot demonstrates the estimated odds ratio (OR) for the correlation of LOS with hospitalized COVID-19 cases for each analysis (grey boxes), with a 95\% confidence interval (CI; horizontal black lines). The pooled OR (grey diamond) was estimated to be 0.283 (95\% CI 0.173-0.462). A two-sided test confirmed that the estimated pooled OR was significantly different from $1(p<0.001)$. Effects and summaries were calculated using a random-effects model weighted by the study population

sustentacular cells or cause loss of cilia, thereby prompting LOS (Butowt and von Bartheld, 2020).

Patients with mild COVID-19 showed early viral clearance compared with severely ill patients (Liu et al. 2020). Severely ill COVID-19 patients demonstrated higher levels of cytokines than non-severely ill COVID-19 patients (McElvaney et al. 2020). It was reported that patients with anosmia presented lower serum cytokine levels and chest computed tomography scans were more subtle and showed milder disease, with a lower progression rate and quicker radiological recovery compared with those without anosmia. (Sanli et al. 2020) The authors also hypothesized that the olfactory epithelium is the first line of defence against viruses, and patients who could generate an antiviral response at the olfactory epithelium may have a milder disease but experience LOS (Sanli et al. 2020). This form of nasal defence mechanism may prevent virus replication and propagation into the lower respiratory system (Gallo et al. 2020; Matricardi et al. 2020).

Nasal mucociliary clearance time was prolonged in hospitalized COVID-19 patients without chemosensory deficits than in healthy otolaryngology outpatients with non-nasal symptoms (Koparal et al. (2020)). Impaired mucociliary clearance may promote SARS-CoV-2 spread into the deeper lung parenchyma (Robinot et al. 2020). Nasal mucociliary clearance, which is a form of nasal defence mechanism, propels the overlying mucus admixed with foreign entities from the airways to the oropharynx where it is either swallowed or expectorated to protect the individual from a lower respiratory tract infection (Chilvers and O'Callaghan 2000). Thus, 
there may be a correlation between anosmia and mucociliary clearance time, which may cause variations in COVID-19 severity; this needs further investigation.

Respiratory neurotropic viruses can invade the central nervous system (CNS) from the nasal cavity via ORN or channels formed by olfactory ensheathing cells across the cribriform plate (van Riel et al. 2015). In contrast, virus induced ORN apoptosis could constitute a neuroprotective feature by blocking the entry of virus to the olfactory bulb and CNS (Mori et al. 2002). SARS-CoV-2 neuroinvasion might infect the respiratory centre of the brain causing the respiratory breakdown of patients with COVID-19 (Gandhi et al. 2020b; Machado et al. 2020). Thus, anosmia in patients with mild disease may indicate a lower chance of SARS-CoV-2 transfer to the CNS; consequently, these patients experience non-severe respiratory and neurological manifestations. Figure 5 demonstrates the possible mechanism behind the correlation between COVID-19 severity and LOS, that could explain why patients without anosmia can develop severe forms of COVID-19.

Several new variants of SARS-CoV-2 have been discovered, which might be more contagious, have serious effects, pose a possible diagnostic risk and current vaccines may not offer overall protection against it (Boehm et al. 2021). The severity of disease in patients with mild symptoms of COVID-19 could progress in approximately a week (Huang et al. 2020). In contrast, most patients experience anosmia within one week, and clinical improvement may occur in the following weeks (Santos et al. 2021). Therefore, early prediction of COVID-19 severity is crucial. A large-scale meta-analysis reported that despite the similar proportion of sex distribution, male patients have approximately three times higher odds of requiring ICU admission and deaths than female patients (Peckham et al. 2020). It was reported that elderly males over 80 years old with comorbidities are more likely to progress to critically ill condition (Meng et al. 2020b). On the other hand, anosmia is reported to be more frequent in females (Talavera et al. 2020). Thus, from our findings, we recommend close monitoring of patients who do not experience anosmia symptom, particularly those at high risk of disease progression such as elderly males with comorbidities, during the first week of COVID-19 symptom onset for early diagnosis of severity of illness. A meta-analysis demonstrated that initial COVID-19 symptoms such as fever, cough, dyspnea, diarrhoea, abdominal pain, anorexia, and fatigue are more frequently seen in severe COVID-19 patients compared to mild patients (He et al. 2021). Our finding strongly suggests anosmia, along with other potential parameters could be a potential factor for predicting early COVID-19 severity, thereby facilitating early intervention and rational distribution of resources.

Since most of the studies asked about changes in chemosensory perception, subjects with pre-existing LOS would generally not have been included, and some studies specifically excluded patients with pre-existing OD; therefore, studies would not have given false positives.

In conclusion, our study shows that severely ill or hospitalized COVID-19 patients are less likely to develop anosmia than non-severely ill or non-hospitalized COVID-19 patients. Several meta-analyses demonstrated the prevalence of anosmia in COVID-19 patients; however, they were unable to assess the association of OD with COVID-19 severity (severe versus non-severe forms). While we attempted to include only objective evaluation data on LOS, only few studies have conducted objective olfactory evaluations characterized by the severity of COVID-19; thus, our study also includes subjective olfactory data. Further investigation of more studies with a large number of participants using only olfactory evaluation data could be considered to validate the findings of current meta-analysis. 

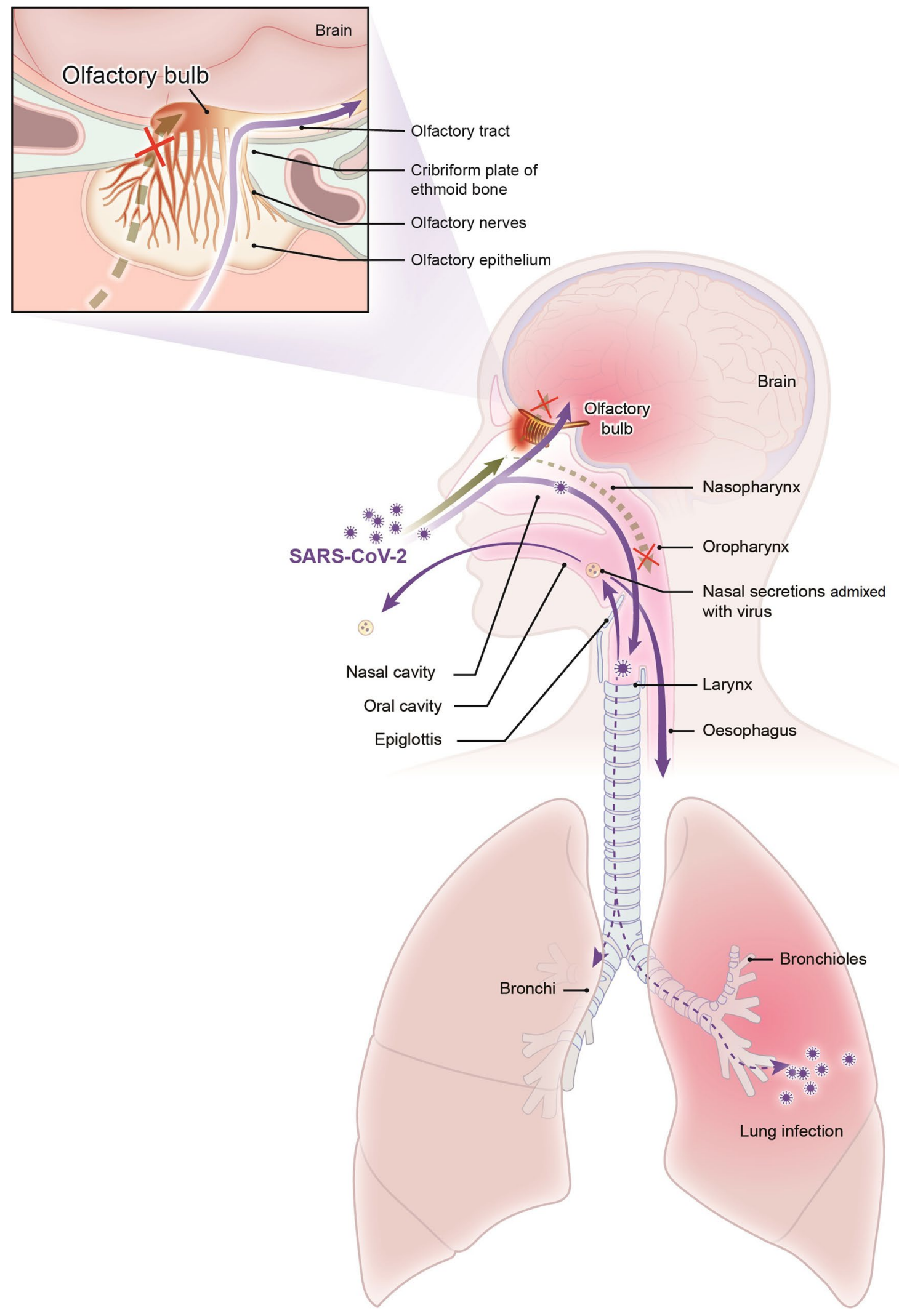
\Fig. 5 The possible mechanism underlying the association of COVID-19 severity with anosmia. SARS-CoV-2 can invade the central nervous system (CNS) via the olfactory pathway (straight purple line leading up towards the brain) or spread to the lower respiratory tract via inhalation after entering the nasal cavity (straight purple line pointing downward). However, viral invasion activates the host immune system and recruits inflammatory mediators, which can cause damage to the olfactory epithelium (red spot), leading to anosmia. This process can prevent viral entry into the CNS by blocking its transmission to the olfactory bulb (green dotted line pointing towards the brain), thereby prevents the infection of respiratory centres in the brain. When this innate immune response is triggered, it can destroy the virus and limit viral propagation to the lower respiratory tract (green dotted line pointing towards the lower respiratory tract). Mucociliary clearance is another nasal defence mechanism that clears the particles that enter the lower respiratory tract by expelling them into the oropharynx from where they are either expectorated or swallowed (straight purple lines pointing toward the oral cavity and oesophagus). However, individuals with risk factors may have compromised nasal defences mechanism, allowing the virus to enter the lower respiratory tract through aspiration into the lungs, resulting in lower respiratory tract infection (dotted purple lines)

Acknowledgements This research was supported by a grant from National Research Foundation of Korea (NRF) grant funded by the Korean government (MSIP) (NRF-2015R1A5A1008958), by Basic Science Research Program through the National Research Foundation of Korea (NRF) funded by the Ministry of Education (2018R1D1A1B07046564), by NRF grant funded by the Korea government (Ministry of Science and ICT, MICT)(NRF2021R1F1A1062044), and by Basic Science Research Program through the National Research Foundation of Korea (NRF) funded by the Ministry of Education (Grant Number 2021R1A6A1A03044296), which had no further role in study design, the collection, analysis, and interpretation of data, the writing of the report, or in the decision to submit the paper for publication.

\section{Declarations}

Conflict of interest The authors declare that they have no conflicts of interests.

\section{References}

Aggarwal S, Garcia-Telles N, Aggarwal G, Lavie C, Lippi G, Henry BM (2020) Clinical features, laboratory characteristics, and outcomes of patients hospitalized with coronavirus disease 2019 (COVID-19): Early report from the United States. Diagnosis (berl) 7:91-96. https://doi.org/10.1515/dx-2020-0046

Al Harthi S, Magdi A, Al Ruwaida I, Al Sultan L, Bina K, Al Mustafa S, Al Nasser K, Al Salim Q, Al Mohamed H, Al Hamad H, Al Thamra G (2020) Clinical characteristics of confirmed cases of COVID-19 admitted at Al-Nahdha hospital, Oman: a cross-sectional descriptive study. Preprint at https://doi.org/10. 21203/rs.3.rs-39988/v1

Alasia D, Owhonda G, Maduka O, Nwadiuto I, Arugu G, Tobin-West C, Azi E, Oris-Onyiri V, Urang IJ, Abikor V, Olofinuka AM, Adebiyi O, Somiari A, Avundaa H, Alali A (2021) Clinical and epidemiological characteristics of 646 hospitalised SARSCov-2 positive patients in Rivers State Nigeria: a prospective observational study. Pan Afr Med J 38:25. https://doi.org/10. 11604/pamj.2021.38.25.26755

Alizadehsani R, Alizadeh Sani Z, Behjati M, Roshanzamir Z, Hussain S, Abedini N, Hasanzadeh F, Khosravi A, Shoeibi A, Roshanzamir M, Moradnejad P, Nahavandi S, Khozeimeh F, Zare A, Panahiazar M, Acharya UR, Islam SMS (2021) Risk factors prediction, clinical outcomes, and mortality in COVID19 patients. J Med Virol 93:2307-2320. https://doi.org/10.1002/ jmv. 26699

Allenbach Y, Saadoun D, Maalouf G, Vieira M, Hellio A, Boddaert J, Gros H, Salem JE, Resche Rigon M, Menyssa C, Biard L, Benveniste O, Cacoub P (2020) Development of a multivariate prediction model of intensive care unit transfer or death: a French prospective cohort study of hospitalized COVID-19 patients. PLoS ONE 15:e0240711. https://doi.org/10.1371/journal.pone.0240711

Amanat M, Rezaei N, Roozbeh M, Shojaei M, Tafakhori A, Zoghi A, Darazam IA, Salehi M, Karimialavijeh E, Lima BS, Garakani A, Vaccaro A, Ramezani M (2021) Neurological manifestations as the predictors of severity and mortality in hospitalized individuals with COVID-19: a multicenter prospective clinical study. BMC Neurol 21:116. https://doi.org/10.1186/s12883-021-02152-5

Amer MA, Elsherif HS, Abdel-Hamid AS, Elzayat S (2020) Early recovery patterns of olfactory disorders in COVID-19 patients; a clinical cohort study. Am J Otolaryngol 41:102725. https:// doi.org/10.1016/j.amjoto.2020.102725

Avcı H, Karabulut B, Farasoglu A, Boldaz E, Evman M (2020) Relationship between anosmia and hospitalisation in patients with coronavirus disease 2019: an otolaryngological perspective. J Laryngol Otol 134:710-716. https://doi.org/10.1017/S0022 215120001851

Aziz M, Goyal H, Haghbin H, Lee-Smith WM, Gajendran M, Perisetti A (2021) The association of "Loss of Smell" to COVID-19: a systematic review and meta-analysis. Am J Med Sci 361:216225. https://doi.org/10.1016/j.amjms.2020.09.017

Baj J, Karakuła-Juchnowicz H, Teresiński G, Buszewicz G, Ciesielka M, Sitarz E, Forma A, Karakuła K, Flieger W, Portincasa P, Maciejewski R (2020) COVID-19: specific and non-specific clinical manifestations and symptoms: the current state of knowledge. J Clin Med 9:1753. https://doi.org/10.3390/jcm90 61753

Bakhshaee M, Barzegar-Amini M, Motedayen Z, Khojasteh-Taheri R, Rafiee M, Amini M, Layegh P, Hashemzadeh K, Omidvar D, Hwang PH, Hosseinpoor M (2021) Olfactory dysfunction in patients infected with 2019 novel coronavirus. Iran J Otorhinolaryngol 33:163-171. https://doi.org/10.22038/ijorl.2021. 51614.2750

Bertlich M, Stihl C, Weiss BG, Canis M, Haubner F, Ihler F (2020) Characteristics of impaired chemosensory function in hospitalized COVID-19 patients. Preprint at https://doi.org/10.2139/ssrn. 3576889

Bianco MR, Modica DM, Drago GD, Azzolina A, Mattina G, De Natale M, Rossi G, Amata M, Canzoneri G, Manganaro G, Di Gregorio F, Russo R, Pricoco S, Saita V, Allegra E (2021) Alteration of smell and taste in asymptomatic and symptomatic COVID19 patients in sicily Italy. Ear Nose Throat J 100:182s-185s. https://doi.org/10.1177/0145561320981447

Boehm E, Kronig I, Neher RA, Eckerle I, Vetter P, Kaiser L (2021) Novel SARS-CoV-2 variants: the pandemics within the pandemic. Clin Microbiol Infect. https://doi.org/10.1016/j.cmi.2021.05.022

Borobia AM, Carcas AJ, Arnalich F, Ãlvarez-Sala R, Monserrat-Villatoro J, Quintana M, Figueira JC, Torres Santos-Olmo RM, GarcíaRodríguez J, Martín-Vega A, Buño A, Ramírez E, Martínez-Alés G, García-Arenzana N, Núñez MC, Martí-De-Gracia M, Moreno Ramos F, Reinoso-Barbero F, Martin-Quiros A, Rivera Núñez A, Mingorance J, Carpio Segura CJ, Prieto Arribas D, Rey Cuevas E, Prados Sánchez C, Rios JJ, Hernán MA, Frías J, Arribas JR, 
and Group, O. B. O. T. C. H. W. A (2020) Cohort of patients with COVID-19 in a major teaching hospital in Europe. J Clin Med 9:1733. https://doi.org/10.3390/jcm9061733

Butowt R, Von Bartheld CS (2020) Anosmia in COVID-19: underlying mechanisms and assessment of an olfactory route to brain infection. Neuroscientist. https://doi.org/10.1177/1073858420956905

Chilvers MA, O'callaghan MA (2000) Local mucociliary defence mechanisms. Paediatr Respir Rev 1:27-34. https://doi.org/10. 1053/prrv.2000.0009

D'ascanio L, Pandolfini M, Cingolani C, Latini G, Gradoni P, Capalbo M, Frausini G, Maranzano M, Brenner MJ, Di Stadio A (2021) Olfactory dysfunction in COVID-19 patients: prevalence and prognosis for recovering sense of smell. Otolaryngol. Head Neck Surg., 164, 82-86. doi: https://doi.org/10.1177/0194599820 943530

Delorme, C., Houot, M., Rosso, C., Carvalho, S., Nedelec, T., Maatoug, R., Pitron, V., Gassama, S., Sambin, S., Bombois, S., Herlin, B., Ouvrard, G., Bruneteau, G., Hesters, A., Gales, A. Z., Millet, B., Lamari, F., Lehericy, S., Navarro, V., Rohaut, B., Demeret, S., Maisonobe, T., Yger, M., Degos, B., Mariani, L.-L., Bouche, C., Dzierzynski, N., Oquendo, B., Ketz, F., Nguyen, A.-H., Kas, A., Lubetzki, C., Delattre, J.-Y., Corvol, J.-C., and Group, C.-N. S (2021) The wide spectrum of Covid-19 neuropsychiatric complications within a multidisciplinary center. Brain Commun. https:// doi.org/10.1093/braincomms/fcab135

Egger M, Davey Smith G, Schneider M, Minder C (1997) Bias in metaanalysis detected by a simple, graphical test. BMJ 315:629-634. https://doi.org/10.1136/bmj.315.7109.629

Elimian KO, Ochu CL, Ebhodaghe B, Myles P, Crawford EE, Igumbor E, Ukponu W, Olayinka A, Aruna O, Dan-Nwafor C, Olawepo OA, Ogunbode O, Atteh R, Nwachukwu W, Venkatesan S, Obagha C, Ngishe S, Suleiman K, Usman M, Yusuff HA, Nwadiuto I, Mohammed AA, Usman R, Mba N, Aderinola O, Ilori E, Oladejo J, Abubakar I, Ihekweazu C (2020) Patient characteristics associated with COVID-19 positivity and fatality in Nigeria: retrospective cohort study. BMJ Open 10:e044079. https://doi.org/10.1136/ bmjopen-2020-044079

Ermis U, Rust MI, Bungenberg J, Costa A, Dreher M, Balfanz P, Marx G, Wiesmann M, Reetz K, Tauber SC, Schulz JB (2021) Neurological symptoms in COVID-19: a cross-sectional monocentric study of hospitalized patients. Neurol Res Pract 3:17. https://doi. org/10.1186/s42466-021-00116-1

Gallo O, Locatello LG, Mazzoni A, Novelli L, Annunziato F (2020) The central role of the nasal microenvironment in the transmission, modulation, and clinical progression of SARS-CoV-2 infection. Mucosal Immunol 14:305-316. https://doi.org/10.1038/ s41385-020-00359-2

Gandhi RT, Lynch JB, Del Rio C (2020a) Mild or Moderate Covid-19. N Engl J Med 383:1757-1766. https://doi.org/10.1056/NEJMc p2009249

Gandhi S, Srivastava AK, Ray U, Tripathi PP (2020b) Is the collapse of the respiratory center in the brain responsible for respiratory breakdown in COVID-19 patients? ACS Chem Neurosci 11:13791381. https://doi.org/10.1021/acschemneuro.0c00217

García-Azorín D, Abildúa MJA, Aguirre MEE, Fernández SF, Moncó JCG, Guijarro-Castro C, Platas MG, Delgado FR, Andrés JML, Ezpeleta D (2021) Neurological presentations of COVID-19: findings from the Spanish Society of Neurology neuroCOVID-19 registry. J Neurol Sci 423:117283. https://doi.org/10.1016/j.jns. 2020.117283

Ghaffari M, Ansari H, Beladimoghadam N, Aghamiri SH, Haghighi M, Nabavi M, Mansouri B, Mehrpour M, Assarzadegan F, Hesami O, Sedaghat M, Farahbakhsh M, Lima BS (2021) Neurological features and outcome in COVID-19: dementia can predict severe disease. J Neurovirol 27:86-93. https://doi.org/10.1007/ s13365-020-00918-0
Goyal R, Kapoor A, Goyal MK, Singh R (2021) Alteration of smell and taste sensations in Covid-19 positive patients: a prospective cohort study in Western India. Indian J Otolaryngol Head Neck Surg. https://doi.org/10.1007/s12070-021-02670-1

Hajikhani B, Calcagno T, Nasiri MJ, Jamshidi P, Dadashi M, Goudarzi M, Eshraghi AA, Mirsaeidi M (2020) Olfactory and gustatory dysfunction in COVID-19 patients: a meta-analysis study. Physiol Rep 8:e14578. https://doi.org/10.14814/phy2.14578

Hannum ME, Ramirez VA, Lipson SJ, Herriman RD, Toskala AK, Lin C, Joseph PV, Reed DR (2020) Objective sensory testing methods reveal a higher prevalence of olfactory loss in COVID-19-positive patients compared to subjective methods: a systematic review and meta-analysis. Chem Senses 45:865-874. https://doi.org/10.1093/ chemse/bjaa064

He X, Cheng X, Feng X, Wan H, Chen S, Xiong M (2021) Clinical symptom differences between mild and severe COVID-19 patients in China: a meta-analysis. Front Public Health 8:561264-561264. https://doi.org/10.3389/fpubh.2020.561264

Higgins JP, Thompson SG, Deeks JJ, Altman DG (2003) Measuring inconsistency in meta-analyses. BMJ 327:557-560. https://doi. org/10.1136/bmj.327.7414.557

Huang C, Wang Y, Li X, Ren L, Zhao J, Hu Y, Zhang L, Fan G, Xu J, Gu X, Cheng Z, Yu T, Xia J, Wei Y, Wu W, Xie X, Yin W, Li H, Liu M, Xiao Y, Gao H, Guo L, Xie J, Wang G, Jiang R, Gao Z, Jin Q, Wang J, Cao B (2020) Clinical features of patients infected with 2019 novel coronavirus in Wuhan, China. Lancet 395:497-506. https://doi.org/10.1016/S0140-6736(20)30183-5

Izquierdo-Domínguez A, Rojas-Lechuga MJ, Chiesa-Estomba C, Calvo-Henríquez C, Ninchritz-Becerra E, Soriano-Reixach M, Poletti-Serafini D, Maria Villarreal I, Maza-Solano JM, MorenoLuna R, Villarroel PP, Mateos-Serrano B, Agudelo D, Valcarcel F, Del Cuvillo A, Santamaría A, Mariño-Sánchez F, Aguilar J, Vergés P, Inciarte A, Soriano A, Mullol J, Alobid I (2020) Smell and taste dysfunction in covid-19 is associated with younger age in ambulatory settings: a multicenter cross-sectional study. $\mathbf{J}$ Investig Allergol Clin Immunol 30:346-357. https://doi.org/10. 18176/jiaci.0595

Izquierdo JL, Ancochea J, Soriano JB (2020) Clinical characteristics and prognostic factors for intensive care unit admission of patients with COVID-19: retrospective study using machine learning and natural language processing. J Med Internet Res 22:e21801. https://doi.org/10.2196/21801

Kadiane-Oussou NJ, Klopfenstein T, Royer PY, Toko L, Gendrin V, Zayet S (2020) COVID-19: comparative clinical features and outcome in 114 patients with or without pneumonia (Nord FrancheComte Hospital, France). Microbes Infect 22:622-625. https://doi. org/10.1016/j.micinf.2020.10.002

Killerby ME, Link-Gelles R, Haight SC, Schrodt CA, England L, Gomes DJ, Shamout M, Pettrone K, O'laughlin, K., Kimball, A., Blau, E. F., Burnett, E., Ladva, C. N., Szablewski, C. M., TobinD'angelo, M., Oosmanally, N., Drenzek, C., Murphy, D. J., Blum, J. M., Hollberg, J., Lefkove, B., Brown, F. W., Shimabukuro, T., Midgley, C. M., and Tate, J. E. (2020) Characteristics associated with hospitalization among patients with COVID-19-Metropolitan Atlanta, Georgia, March-April 2020. MMWR Morb Mortal Wkly Rep 69:790-794. https://doi.org/10.15585/mmwr.mm6925e1

Kocayı ğıt H, Øzmen Süner K, Tomak Y, Demır G, Kocayı ğıt İ, Yaylaci S, Aydemır Y, Köroğlu M, Karabay O, Øztürk MH, Erdem AF (2021) Characteristics and outcomes of critically ill patients with covid-19 in Sakarya, Turkey: a single centre cohort study. Turk J Med Sci 51:440-447. https://doi.org/10.3906/sag-2005-57

Koparal M, Kurt E, Altuntas EE, Dogan F (2020) Assessment of mucociliary clearance as an indicator of nasal function in patients with COVID-19: a cross-sectional study. Eur Arch Otorhinolaryngol. https://doi.org/10.1007/s00405-020-06457-y 
Lechien JR, Chiesa-Estomba CM, Beckers E, Mustin V, Ducarme M, Journe F, Marchant A, Jouffe L, Barillari MR, Cammaroto G, Circiu MP, Hans S, Saussez S (2021) Prevalence and 6-month recovery of olfactory dysfunction: a multicentre study of 1363 COVID-19 patients. J Intern Med. https://doi.org/10.1111/joim. 13209

Liang Y, Xu J, Chu M, Mai J, Lai N, Tang W, Yang T, Zhang S, Guan C, Zhong F, Yang L, Liao G (2020) Neurosensory dysfunction: a diagnostic marker of early COVID-19. Int J Infect Dis 98:347352. https://doi.org/10.1016/j.ijid.2020.06.086

Liotta EM, Batra A, Clark JR, Shlobin NA, Hoffman SC, Orban ZS, Koralnik IJ (2020) Frequent neurologic manifestations and encephalopathy-associated morbidity in Covid-19 patients. Ann Clin Transl Neurol 7:2221-2230. https://doi.org/10.1002/acn3. 51210

Liu Y, Yan L-M, Wan L, Xiang T-X, Le A, Liu J-M, Peiris M, Poon LLM, Zhang W (2020) Viral dynamics in mild and severe cases of COVID-19. Lancet Infect Dis 20:656-657. https://doi.org/10. 1016/S1473-3099(20)30232-2

Machado C, Defina PA, Chinchilla M, Machado Y, Machado Y (2020) Brainstem dysfunction in SARS-COV-2 infection can be a potential cause of respiratory distress. Neurol India 68:989-993. https:// doi.org/10.4103/0028-3886.299165

Mao L, Jin H, Wang M, Hu Y, Chen S, He Q, Chang J, Hong C, Zhou Y, Wang D, Miao X, Li Y, Hu B (2020) Neurologic manifestations of hospitalized patients with coronavirus disease 2019 in Wuhan China. JAMA Neurol 77:683-690. https://doi.org/10.1001/jaman eurol.2020.1127

Matricardi PM, Dal Negro RW, Nisini R (2020) The first, holistic immunological model of COVID-19: implications for prevention, diagnosis, and public health measures. Pediatr Allergy Immunol 31:454-470. https://doi.org/10.1111/pai.13271

Mcelvaney OJ, Mcevoy NL, Mcelvaney OF, Carroll TP, Murphy MP, Dunlea DM, Ní Choileáin O, Clarke J, O'connor, E., Hogan, G., Ryan, D., Sulaiman, I., Gunaratnam, C., Branagan, P., O'brien, M. E., Morgan, R. K., Costello, R. W., Hurley, K., Walsh, S., De Barra, E., Mcnally, C., Mcconkey, S., Boland, F., Galvin, S., Kiernan, F., O'rourke, J., Dwyer, R., Power, M., Geoghegan, P., Larkin, C., O'leary, R. A., Freeman, J., Gaffney, A., Marsh, B., Curley, G. F., and Mcelvaney, N. G. (2020) Characterization of the inflammatory response to severe COVID-19 illness. Am J Respir Crit Care Med 202:812-821. https://doi.org/10.1164/rccm. 202005-1583OC

Mendonça CV, Mendes Neto JA, Suzuki FA, Orth MS, Machado Neto H, Nacif SR (2021) Olfactory dysfunction in COVID-19: a marker of good prognosis? Braz J Otorhinolaryngol S18088694(20):30240-30248. https://doi.org/10.1016/j.bjorl.2020.12. 002

Meng X, Deng Y, Dai Z, Meng Z (2020a) COVID-19 and anosmia: a review based on up-to-date knowledge. Am J Otolaryngol 41:102581-102581. https://doi.org/10.1016/j.amjoto.2020.102581

Meng Y, Wu P, Lu W, Liu K, Ma K, Huang L, Cai J, Zhang H, Qin Y, Sun H, Ding W, Gui L, Wu P (2020b) Sex-specific clinical characteristics and prognosis of coronavirus disease-19 infection in Wuhan, China: A retrospective study of 168 severe patients. PLoS Pathog 16:e1008520. https://doi.org/10.1371/journal.ppat. 1008520

Mori I, Goshima F, Imai Y, Kohsaka S, Sugiyama T, Yoshida T, Yokochi T, Nishiyama Y, Kimura Y (2002) Olfactory receptor neurons prevent dissemination of neurovirulent influenza A virus into the brain by undergoing virus-induced apoptosis. J Gen Virol 83:2109-2116. https://doi.org/10.1099/0022-1317-83-9-2109

Moola S, Munn Z, Tufanaru C, Aromataris E, Sears K, Sfetcu R, Currie M, Lisy K, Qureshi R, Mattis P, Mu P (2020) Chapter 7: Systematic reviews of etiology and risk. In: Aromataris E, Munn Z (eds) JBI Manual for Evidence Synthesis, JBI. Available from https://synthesismanual.jbi.global. https://doi.org/10.46658/ JBIMES-20-08

Muñoz-Rodríguez JR, Gómez-Romero FJ, Pérez-Ortiz JM, LópezJuárez P, Santiago JL, Serrano-Oviedo L, Redondo-Calvo FJ (2021) Characteristics and risk factors associated with mortality in a multicenter spanish cohort of patients with COVID-19 pneumonia. Arch Bronconeumol 57(2):34-41. https://doi.org/10. 1016/j.arbres.2021.02.021

Nouchi A, Chastang J, Miyara M, Lejeune J, Soares A, Ibanez G, Saadoun D, Morélot-Panzini C, Similowski T, Amoura Z, Boddaert J, Caumes E, Bleibtreu A, Lorenzo A, Tubach F, Pourcher V (2021) Prevalence of hyposmia and hypogeusia in 390 COVID-19 hospitalized patients and outpatients: a cross-sectional study. Eur J Clin Microbiol Infect Dis 40:691-697. https://doi.org/10.1007/ s10096-020-04056-7

Paderno A, Schreiber A, Grammatica A, Raffetti E, Tomasoni M, Gualtieri T, Taboni S, Zorzi S, Lombardi D, Deganello A, Redaelli De Zinis LO, Maroldi R, Mattavelli D (2020) Smell and taste alterations in COVID-19: a cross-sectional analysis of different cohorts. Int Forum Allergy Rhinol 10:955-962. https://doi.org/ 10.1002/alr.22610

Papizadeh S, Moradi P, Mehr MH, Amerkani S, Nezhad RF, Saadati H, Shahani T, Mohammadian M, Sadooghi N, Bahari S, Ghorbani A, Mehrabi M, Farzi R, Ranjbar R, Ghorbani S (2020) Epidemiologic and clinical characteristics of 186 hospitalized patients with Covid-19 in Tehran, Iran: a retrospective, single-center case series. Preprint at https://www.preprints.org/manuscript/202007. 0060/v1

Patel A, Bhatt P, Madan S, Shah N, Thakkar V, Shah B, Chovatia R, Shah H, Patel M, Dabhi P, Nanavati A, Chag M, Parikh K (2020) A comparative COVID 19 characterizations and clinical course analysis between ICU and non ICU settings. Preprint at https:// doi.org/10.1101/2020.10.07.20208389

Peckham H, De Gruijter NM, Raine C, Radziszewska A, Ciurtin C, Wedderburn LR, Rosser EC, Webb K, Deakin CT (2020) Male sex identified by global COVID-19 meta-analysis as a risk factor for death and ITU admission. Nat Commun 11:6317. https://doi. org/10.1038/s41467-020-19741-6

Printza A, Katotomichelakis M, Valsamidis K, Metallidis S, Panagopoulos P, Panopoulou M, Petrakis V, Constantinidis J (2021) Smell and taste loss recovery time in COVID-19 patients and disease severity. J Clin Med. https://doi.org/10.3390/jcm10050966

Robinot R, Hubert M, De Melo GD, Lazarini F, Bruel T, Smith N, Levallois S, Larrous F, Fernandes J, Gellenoncourt S, Rigaud S, Gorgette O, Thouvenot C, Trébeau C, Mallet A, Duménil G, Gobaa S, Etournay R, Lledo P-M, Lecuit M, Bourhy H, Duffy D, Michel V, Schwartz O, Chakrabarti LA (2020) SARS-CoV-2 infection damages airway motile cilia and impairs mucociliary clearance. Preprint at https://doi.org/10.1101/2020.10.06.328369

Rodriguez S, Cao L, Rickenbacher GT, Benz EG, Magdamo C, Gomez LAR, Holbrook E, Albers AD, Gallagher R, Brandon Westover M, Evans KE, Tatar D, Mukerji S, Zafonte R, Boyer EW, Ron Yu C, Albers MW (2020) Innate immune signaling in the olfactory epithelium reduces odorant receptor levels: modeling transient smell loss in COVID-19 patients. Preprint at https://doi.org/10. 1101/2020.06.14.20131128

Romero-Sánchez CM, Díaz-Maroto I, Fernández-Díaz E, SánchezLarsen Ã, Layos-Romero A, García-García J, González E, Redondo-Peñas I, Perona-Moratalla AB, Del Valle-Pérez JA, Gracia-Gil J, Rojas-Bartolomé L, Feria-Vilar I, Monteagudo M, Palao M, Palazón-García E, Alcahut-Rodríguez C, Sopelana-Garay D, Moreno Y, Ahmad J, Segura T (2020) Neurologic manifestations in hospitalized patients with COVID-19: The ALBACOVID registry. Neurology 95:e1060-e1070. https://doi.org/10.1212/WNL. 0000000000009937 
Salepci E, Turk B, Ozcan SN, Bektas ME, Aybal A, Dokmetas I, Turgut S (2021) Symptomatology of COVID-19 from the otorhinolaryngology perspective: a survey of 223 SARS-CoV-2 RNA-positive patients. Eur Arch Otorhinolaryngol 278:525-535. https://doi.org/ 10.1007/s00405-020-06284-1

Sanli DET, Altundag A, Kandemirli SG, Yildirim D, Sanli AN, Saatci O, Kirisoglu CE, Dikensoy O, Murrja E, Yesil A, Bastan S, Karsidag T, Akinci IO, Ozkok S, Yilmaz E, Tuzuner F, Kilercik M, Ljama T (2020) Relationship between disease severity and serum IL levels in COVID anosmia. Am J Otolaryngol. https://doi.org/ 10.1016/j.amjoto.2020.102796

Santos REA, Da Silva MG, do Monte Silva MC, Barbosa, D. A. M., Gomes, A. L. D. V., Galindo, L. C. M., Da Silva Aragão, R., and Ferraz-Pereira, K. N. (2021) Onset and duration of symptoms of loss of smell/taste in patients with COVID-19: a systematic review. Am J Otolaryngol 42:102889-102889. https://doi.org/10. 1016/j.amjoto.2020.102889

Sepahi A, Kraus A, Casadei E, Johnston CA, Galindo-Villegas J, Kelly C, García-Moreno D, Muñoz P, Mulero V, Huertas M, Salinas I (2019) Olfactory sensory neurons mediate ultrarapid antiviral immune responses in a TrkA-dependent manner. Proc Natl Acad Sci U S A 116:12428-12436. https://doi.org/10.1073/pnas.19000 83116

Sobhani S, Aryan R, Kalantari E, Soltani S, Malek N, Pirzadeh P, Yarahmadi A, Aghaee A (2021) Association between clinical characteristics and laboratory findings with outcome of hospitalized COVID-19 patients: a report from Northeast Iran. Interdiscip Perspect Infect Dis 2021:5552138. https://doi.org/10.1155/2021/ 5552138

Song J, Deng Y-K, Wang H, Wang Z-C, Liao B, Ma J, He C, Pan L, Liu Y, Alobid I, Wang D-Y, Zeng M, Mullol J, Liu Z (2021) Selfreported taste and smell disorders in patients with COVID-19: distinct features in China. Curr Med Sci 41:14-23. https://doi.org/ 10.1007/s11596-021-2312-7

Studart-Neto A, Guedes BF, Tuma RLE, Camelo Filho AE, Kubota GT, Iepsen BD, Moreira GP, Rodrigues JC, Ferrari MMH, Carra RB, Spera RR, Oku MHM, Terrim S, Lopes CCB, Passos Neto CEB, Fiorentino MD, Jcc DES, Baima JPS, Tff D. A. S., Moreno, C. A. M., Silva, A. M. S., Heise, C. O., MendonÉa, R. H., Fortini, I., Smid, J., Adoni, T., GonÉalves, M. R. R., Pereira, S. L. A., Pinto, L. F., Gomes, H. R., Zanoteli, E., Brucki, S. M. D., Conforto, A. B., Castro, L. H. M., and Nitrini, R. (2020) Neurological consultations and diagnoses in a large, dedicated COVID-19 university hospital. Arq Neuropsiquiatr 78:494-500. https://doi.org/10.1590/ 0004-282x20200089

Sun LL, Wang J, Wang YS, Hu PF, Zhao ZQ, Chen W, Ning BF, Yin C, Hao YS, Wang Q, Wang C, Liu YL, Chen C, Yin JZ, Huang H, Xie WF (2021) Symptomatic features and prognosis of 932 hospitalized patients with coronavirus disease 2019 in Wuhan. J Dig Dis 22:271-281. https://doi.org/10.1111/1751-2980.12983

Talavera B, García-Azorín D, Martínez-Pías E, Trigo J, HernándezPérez I, Valle-Peñacoba G, Simón-Campo P, De Lera M, Chavarría-Miranda A, López-Sanz C, Gutiérrez-Sánchez M, MartínezVelasco E, Pedraza M, Sierra Ã, Gómez-Vicente B, Guerrero Ã, Arenillas JF (2020) Anosmia is associated with lower in-hospital mortality in COVID-19. J Neurol Sci. https://doi.org/10.1016/j. jns.2020.117163

Tomlins J, Hamilton F, Gunning S, Sheehy C, Moran E, Macgowan A (2020) Clinical features of 95 sequential hospitalised patients with novel coronavirus 2019 disease (COVID-19), the first UK cohort. J Infect 81:e59-e61. https://doi.org/10.1016/j.jinf.2020.04.020

Tong JY, Wong A, Zhu D, Fastenberg JH, Tham T (2020) The prevalence of olfactory and gustatory dysfunction in COVID-19 patients: a systematic review and meta-analysis. Otolaryngol Head Neck Surg 163:3-11. https://doi.org/10.1177/0194599820926473

Vahey GM, Marshall KE, Mcdonald E, Martin SW, Tate JE, Midgley CM, Killerby ME, Kawasaki B, Herlihy RK, Alden NB, Staples JE (2021) Symptom profiles and progression in hospitalized and nonhospitalized patients with coronavirus disease, Colorado, USA, 2020. Emerg Infect Dis 27:385-395. https://doi.org/10. 3201/eid2702.203729

Vaira LA, Hopkins C, Salzano G, Petrocelli M, Melis A, Cucurullo M, Ferrari M, Gagliardini L, Pipolo C, Deiana G, Fiore V, De Vito A, Turra N, Canu S, Maglio A, Serra A, Bussu F, Madeddu G, Babudieri S, Giuseppe Fois A, Pirina P, Salzano FA, De Riu P, Biglioli F, De Riu G (2020) Olfactory and gustatory function impairment in COVID-19 patients: Italian objective multicenterstudy. Head Neck 42:1560-1569. https://doi.org/10.1002/hed. 26269

Van Riel D, Verdijk R, Kuiken T (2015) The olfactory nerve: a shortcut for influenza and other viral diseases into the central nervous system. J Pathol 235:277-287. https://doi.org/10.1002/path.4461

Vial MR, Peters A, Pérez I, Spencer-Sandino M, Barbé M, Porte L, Weitzel T, Aylwin M, Vial P, Araos R, Munita JM, Marcotti A, Pérez J, Noriega LM, Gaete P, Solar S, López S, Legarraga P, Vollrath V, Anderson A, Iruretagoyena M, Graf J, Pérez R, Roa MA, For The C, A. S. U. D. D. C.-W. G. (2020) Covid-19 in South America: clinical and epidemiological characteristics among 381 patients during the early phase of the pandemic in Santiago. Chile BMC Infect Dis 20:955. https://doi.org/10.1186/ s12879-020-05665-5

Von Bartheld CS, Hagen MM, Butowt R (2020) Prevalence of chemosensory dysfunction in COVID-19 patients: a systematic review and meta-analysis reveals significant ethnic differences. ACS Chem Neurosci 11:2944-2961. https://doi.org/10.1021/acsch emneuro.0c00460

Wells G, Shea B, O'connell D, Peterson J, Welch V, Losos M, Tugwell P (2021) The Newcastle-Ottawa Scale (NOS) for assessing the quality of non-randomized studies in meta-analysis. http://www. ohri.ca/programs/clinical_epidemiology/oxford.asp. Accessed 17 May 2021

Wölfel R, Corman VM, Guggemos W, Seilmaier M, Zange S, Müller MA, Niemeyer D, Jones TC, Vollmar P, Rothe C, Hoelscher M, Bleicker T, Brünink S, Schneider J, Ehmann R, Zwirglmaier K, Drosten C, Wendtner C (2020) Virological assessment of hospitalized patients with COVID-2019. Nature 581:465-469. https://doi. org/10.1038/s41586-020-2196-x

Wu X, Nethery RC, Sabath MB, Braun D, Dominici F (2020) Air pollution and COVID-19 mortality in the United States: strengths and limitations of an ecological regression analysis. Sci Adv 6:4049. https://doi.org/10.1126/sciadv.abd4049

Yan CH, Faraji F, Prajapati DP, Ostrander BT, Deconde AS (2020) Self-reported olfactory loss associates with outpatient clinical course in COVID-19. Int Forum Allergy Rhinol 10:821-831. https://doi.org/10.1002/alr.22592

Zobairy H, Shamsoddin E, Rasouli MA, Khodlan N V, Moradi G, Zareie B, Teymori S, Asadi J, Sofi-Mahmudi A, Sedaghat AR (2020) Association of olfactory dysfunction with hospitalization for COVID-19: a multicenter study in Kurdistan. Preprint at https://doi.org/10.1101/2020.07.26.20158550

Publisher's Note Springer Nature remains neutral with regard to jurisdictional claims in published maps and institutional affiliations. 